\title{
Causes of Osteoporosis in Pediatric Age Group Patients, Following at King Abdul Aziz University Hospital, Jeddah, Saudi Arabia: 12 Years' Experience
}

\author{
Abdulmoein E Al-Agha ${ }^{1 *}$, Alnajjar $\mathrm{FN}^{2}$ and Alkharji $\mathrm{TM}^{3}$ \\ ${ }^{1}$ Faculty of Medicine, King Abdulaziz University, Jeddah, Saudi Arabia \\ ${ }^{2}$ Department of Pediatrics, Faculty of Medicine, King Abdulaziz University, Jeddah, Saudi Arabia \\ ${ }^{3}$ Faculty of Medicine, University of Jeddah, Jeddah, Saudi Arabia
}

"Corresponding author: Abdulmoein E Al-Agha, Associate Professor and Consultant Pediatric Endocrinologist, Department of Pediatrics, King Abdulaziz University Hospital, P.O. Box 80215 Jeddah 21589, Kingdom of Saudi Arabia, Tel: + 966-505590459; E-mail: aagha@kau.edu.sa

Received date: Jan 15, 2016; Accepted date: Feb 17, 2016; Published date: Feb 25, 2016

Copyright: ( 2016 Al-Agha AE, et al. This is an open-access article distributed under the terms of the Creative Commons Attribution License, which permits unrestricted use, distribution, and reproduction in any medium, provided the original author and source are credited.

\begin{abstract}
Background: Osteoporosis in children has various underlying causes related to hereditary or secondary to different diseases like: hematological diseases, gastrointestinal diseases, renal diseases, endocrine diseases, druginduced and immobilization.
\end{abstract}

Objective: To investigate the causes of osteoporosis in pediatrics age group presented to pediatric clinic in King Abdulaziz University Hospital.

Methods: One hundred thirty one children and adolescents with osteoporosis, visiting pediatric clinic at King Abdulaziz University Hospital (KAUH), Jeddah, Saudi Arabia from 2003 to 2015. Seventy five patients had primary osteoporosis which represents $57.3 \%$ of the studied sample of the population. Secondary osteoporosis was found in Fifty six patients which represents $42.7 \%$ of the sample of the population. Various underlying causes of primary and secondary of osteoporosis were evaluated by reviewing medical records file.

Results: The mean age group is 11.43 years. Primary osteoporosis specifically osteogenesis imperfecta accounts for $57.3 \%$ of the cases. Among the secondary cases, hematological diseases, gastrointestinal diseases, endocrine diseases, Renal disease, immobilization, chronic use of steroid, were the most common accounting for $9.2 \%, 9.2 \%, 6.9 \%, 6.1 \%, 6.1 \%, 5.4 \%$ of the cases respectively.

Conclusion: Osteogenesis imperfecta is the most common cause of children osteoporosis at KAUH, Jeddah, Saudi Arabia. While secondary osteoporosis is still to be considered and screened for.

Keywords: Metabolic bone disease; Osteoporosis causes; Children

\section{Introduction}

Osteoporosis is the major health problem all over the world [1] While the osteoporosis is also considered as one of the commonest human metabolic bone disorder in adults, its prevalence in pediatric population is not well studied. In recent years, osteoporosis in children and adolescents has attracted worldwide attention. It is increasingly recognized disease in children and adolescents, and no longer exclusively the inflicted adults and older people. Osteoporosis related fracture is a major public health burden.

In 2007, the international Society of Clinical Densitometry (ISCD) in Montreal Conference has defined pediatric osteoporosis can be diagnosed by pediatric physician clinically in patients with a serious fracture (even with normal investigation result) and age-adjusted Zscores $\leq-2.0$ standard deviation in Dual Energy X-ray absorptiometry (DEXA.). With high bone turnover reflected by CTX and osteocalcin [2].
The causes of osteoporosis in childhood age can be categorized to primary osteoporosis and secondary osteoporosis. The primary type occurs due to an intrinsic bone abnormality that is usually inherited [3], whereas secondary osteoporosis arises from the patient's condition or as a side effect of medication. Based on literature review, the most commonly occurring primary osteoporosis are osteogenesis imperfect [4].

OI is a genetic disorder due to mutation in COL1A1 and COL1A2 in $95 \%$ of OI cases [4], the most common genetic inheritance type is the dominant type, by estimation the incidence rate of (OI) about 1-2 out of 20.000 births per year. Connective tissue disorders like (marfan, Ethlers-danlos) are also at risk of low bone health [4]. Juvenile osteoporosis is usually presented by multiple recurrent fractures, inclusive compression fracture of the back [5]; it is rare form of primary osteoporosis and diagnosis by exclusion [1]. Clinically presentation of OI is the birth fractures as first presentation. Others, including blue sclera, short stature, limp deformity, family history, hyper-laxity of ligaments and hearing impairment [4].

Secondary type of osteoporosis is a significant public health problem in children, and being a silent disease is the most critical 
characteristic of it. The result is that bone loss may not be detected till a fracture occurs [6] or a bone density examination points out that the child or young adult is at serious risk of fracture. A wide spectrum of chronic illness or its medication can lead to secondary type of osteoporosis. Common conditions associated with an increased risk of fracture and osteoporosis in children that includes hematological diseases, endocrine diseases, inflammatory diseases, immobilization $[5,7]$ and a long term use of steroid, which even at low dosages causes a significant decrease in bone mineral density [8].

The objective from this study was to determine the causes and risk factors of osteoporosis in children attending the pediatric clinic at king Abdulaziz University Hospital, Jeddah, Saudi Arabia.

\section{Methodology}

This is a retrospective study conducted on children and adolescents with osteoporosis following up in the pediatric clinic at (KAUH) from January 2003 to January 2015. The study sample of population contains one hundred thirty one children's and adolescents with osteoporosis. Age (0-18) years old. The data was collected in a questionnaire form. This data contains demographic information, anthropometric measurements, history of osteoporosis including age of onset, age at diagnosis, method of diagnosis and the etiology of the disease.

\section{Assessment}

Anthropometric measurements: In this study body height and weight to all the children and adolescents were measured by nurse. For patient aged 2-18 years, age and sex standardized scores (SDs) were measured instead of height and weight of the body, by using Children's Hospital Boston Calculator Growth $2.0[9,10]$.

Clinical assessment: Assessment of the patient condition was completed by a questionnaire filled in by physicians for the patients and/or their parents during the follow-up in the pediatric clinic (KAUH).

Lab assessment: We have measured (25-hydroxyl $(\mathrm{OH})$ vitamin $\mathrm{D}$, Serum calcium, phosphate, alkaline phosphates, parathyroid hormone). Furthermore, the amounts of creatinine were also calculated at baseline, before giving any dose of Zoledronic acid. According to measurements in the study, the normal ranges of: serum calcium was $2.12-2.52 \mathrm{mmol} / \mathrm{L}$. The study categorized hypocalcaemia according to the severity; mild 1.8-2.11, moderate $1.5-1.7$, severe $<1.5$. Hence, 25-hydroxyl $(\mathrm{OH})$ vitamin $\mathrm{D}$ was measured to be $50 \mathrm{nmol} / \mathrm{L}-80$ $\mathrm{nmol} / \mathrm{L}$. Serum Osteocalcin values were normal in females ranged between $11-43 \mathrm{ng} / \mathrm{ml}$, while in males it ranged between $24-70 \mathrm{ng} / \mathrm{ml}$. Serum CTX values in females were less than $0.57 \mathrm{ng} / \mathrm{ml}$, while in males it was less than $0.58 \mathrm{ng} / \mathrm{ml}$. Bone Markers including (serum osteocalcin and CTX).

Radiological assessment: In our study there was some limitation in Bone Mass Density (BMD) assessments because DEXA scan machine in (KAUH) which is not made to measure the children bone density below the 5 years. Because of the malfunction of the machine frequent and some missing results for the baseline DEXA as it was done outside our hospital for many of the patients. We decided not to include any radiological data in this study.

\section{Result}

One hundred thirty one children and adolescents with osteoporosis 67 girls (51.1\%), 64 boys (48.9\%). Mean ages of the sample, i.e., 11.43 years .were included in the study. Patients whose ages were less than 10 years were $48(36.9 \%)$, while who whose ages were more than 10 years were 82 patients (63.1\%). Demographic data of participants are summarized in (Table 1) and secondary causes summarized in (Table 2), Of the 131 patients. Seventy five patients had primary osteoporosis which represents (57.3\%) of the studied sample of population. Secondary osteoporosis was found in Fifty six patients which represents $(42.7 \%)$ of the sample. The commonest cause of primary osteoporosis was osteogenesis imperfecta that accounts for $(55.7 \%)$ of the primary causes and was found to be by far the commonest cause of osteoporosis in general, idiopathic (1.5\%). The commonest cause of secondary osteoporosis was hematological diseases and gastrointestinal diseases which account (9.2\%) each, Endocrine diseases (6.9\%), Renal diseases (6.1\%), Immobilization (6.1\%), Drug induced (5.4\%).

\begin{tabular}{|l|l|l|l|}
\hline \multicolumn{2}{|c|}{} & Frequency & Percentage \\
\hline \multirow{4}{*}{ Primary Osteoporosis } & Osteogenesis imperfecta & 73 & $55.70 \%$ \\
\cline { 2 - 4 } & Idiopathic & 2 & $1.50 \%$ \\
\hline \multirow{5}{*}{ Secondary Osteoporosis } & Hematological diseases & 12 & $9.20 \%$ \\
\cline { 2 - 4 } & Gastrointestinal diseases & 12 & $9.20 \%$ \\
\cline { 2 - 4 } & Endocrine diseases & 9 & $6.90 \%$ \\
\cline { 2 - 4 } & Renal diseases & 8 & $6.10 \%$ \\
\cline { 2 - 4 } & Immobilization & 8 & $6.10 \%$ \\
\cline { 2 - 4 } & Drug induced & 7 & $5.40 \%$ \\
\hline \multirow{2}{*}{ Total } & & 131 & $100 \%$ \\
\hline
\end{tabular}

Table 1: Demographic data of participants. 
Citation: Abdulmoein E Al-Agha1, Alnajjar FN and Alkharji TM (2016) Causes of Osteoporosis in Pediatric Age Group Patients, Following at King Abdul Aziz University Hospital, Jeddah, Saudi Arabia: 12 Years' Experience. J Pat Care 2: 109. doi:10.4172/2573-4598.1000109

Page 3 of 5

\begin{tabular}{|c|c|c|c|}
\hline & & Frequency & Percentage \\
\hline \multirow{2}{*}{ Hematological diseases } & Sickle cell anemia & 8 & $6.10 \%$ \\
\hline & Thalassemia & 4 & $3.10 \%$ \\
\hline \multirow{3}{*}{ Gastrointestinal diseases } & Crohn's disease & 6 & $4.60 \%$ \\
\hline & Ulcerative colitis & 2 & $1.50 \%$ \\
\hline & Celiac disease & 4 & $3.10 \%$ \\
\hline \multirow{2}{*}{ Endocrine diseases } & Insulin dependent diabetes & 8 & $6.10 \%$ \\
\hline & Growth hormone deficiency & 1 & $0.80 \%$ \\
\hline \multirow{3}{*}{ Renal disease } & Hypophosphatemic Rickets & 4 & $3.10 \%$ \\
\hline & Renal failure & 2 & $1.50 \%$ \\
\hline & Renal tubular acidosis & 2 & $1.50 \%$ \\
\hline Immobilization & Immobilization & 8 & $6.10 \%$ \\
\hline \multirow{4}{*}{ Drug induced } & Steroid & 3 & $2.30 \%$ \\
\hline & Steroid + Chemo & 2 & $1.50 \%$ \\
\hline & Methotrexate & 1 & $0.80 \%$ \\
\hline & GnRH agonist & 1 & $0.80 \%$ \\
\hline
\end{tabular}

Table 2: Secondary causes.

\section{Consanguinity}

The Consanguinity is common in our population. Concerning the sample, found in 53 patients (40.5\%).reported in (57.3\%) of the patient with primary osteoporosis, while in secondary that represents $(50 \%)$, 36 patients $(27.5 \%)$ of them were first degree relatives and 16 patients $(12.2 \%)$ were second degree.

\section{Family history}

Thirty one $(23.7 \%)$ of children that had positive family history of osteoporosis before the age of 50, and twenty one (16\%) of children had positive family history of osteoporosis after the age of 50 .

\section{Discussion}

This paper focuses on the causes of osteoporosis in children and adolescent. In our study the sample size is one hundred thirty one children and adolescents with osteoporosis, include 75 (57.3\%) children with primary osteoporosis, and $52(42.7 \%)$ children with secondary osteoporosis. Osteoporosis affects the whole life of the child and his family, and the management and prognosis of the child can be affected by the underlying etiology. Genetic factor is an important risk factor for development of pediatric osteoporosis. According to the present retrospective study primary osteoporosis is the commonest cause of osteoporosis in children and adolescents. Osteogenesis imperfecta specifically was the most prevalent primary etiology presented to our pediatric clinic. By estimation the incidence rate of (OI) about 1-2 out of 20.000 births per year. Comparing to previous across-sectional multicenter study in North America, the prevalence of (OI) 1 in 15.000-20.000 birth [11]. Consanguinity was very prevalent among primary patients and that increases the risk of the offspring being affected with OI and juvenile osteoporosis. The Consanguinity is common in our population. Found that in 53, which represents $(40.5 \%)$ of sample. reported in $(57.3 \%)$ of the patients with primary osteoporosis, while reported in (50\%) in secondary, 36 patient $(27.5 \%)$ of them were first degree relatives and 16 patients (12.2\%) were second degree This finding consistent with previous studies using same method of our data collecting, it has proved that genetic is the most likely risk factor for idiopathic juvenile osteoporosis [1].Thirty one (23.7\%) of children had positive family history of osteoporosis before the age of 50 ,and twenty one (16\%) of children had positive family history of osteoporosis after the age of 50. In North America studies on (544) patient with OI the family history was present in (25\%) [11].

\section{Hematological diseases (HD)}

In our population Hematological disease is considered as one of the most common causes of secondary osteoporosis it accounts for (9.2\%) of the total patient with osteoporosis. Sickle cell anemia is the most common hemolytic disease it accounts for $6.1 \%$ of the total patient with osteoporosis causes which is considered as the most common among the secondary causes. In contrast, comparing to previous reported study in Nicosia, Cyprus [12], 50\% of morbidity from thalassemia major patients suffering from osteoporosis. Patients of our population have found to be thalassemic $3.1 \%$ of the total patient with osteoporosis.

\section{Gastrointestinal diseases}

In our population gastrointestinal disease is considered as one of the most common causes of secondary osteoporosis it presents in (9.2\%) of the total patient with osteoporosis. Comparing to the previous surveys celiac disease affects up to $1 \%$ of the population in the USA and 
Europe [13]. In the other study on 143 gastrointestinal diseases children with (98 with crohns disease, 45 with ulcerative colitis) it is observed: lower height, body weight, muscle mass, total bone cross sectional area, while the recently diagnosed children are with the ratio of bone mineral content / muscle cross sectional area higher than those children with long duration [13]. Reviewing in adult literature on such association found that on USA, in large (9704 subject) study, celiac diseases increase the incidence of bone diseases up to $80 \%$ if untreated and Crohn's disease [14].

\section{Endocrine diseases}

Insulin Dependent Diabetes Mellitus (IDDM) is an important secondary cause of osteoporosis. In our study, it accounts for $5.3 \%$ of the secondary causes which considered as the second most common among the secondary causes. There are 7 diabitiec patients 3 of them have also celiac disease. The fracture risk in children with diabetes there are few data on long term effects. The reported study in Sweden with large diabetic population (24.605) patient 12.551 men and 12.054 Women admission to the hospital for diabetes less than 31 years, registered only 121 first hip fractures. After 40 years of follow up, type 1 Diabetic patients had more than fourfold relative risk of hip fractures with a global accumulative probability of 65.8 / 1000 [13]. Another study consider the children patients with typel diabetes ,had high recurrence of low vitamin $\mathrm{D}$ level, reported also from very sunny countries, in Australia reported 47 children with diabetes aged (12-14 years) $43 \%$ had $\_20 \mathrm{ng} / \mathrm{ml}(50 \mathrm{nmol} / \mathrm{L})$ of serum $25 \mathrm{OHD}$. Another studies in Qatar 170 patients with typel diabetes aged less than 16 years serum 25OHD was 15.8_9.2 ng/ml (39.4_23 nmol/L) on average. Only $16(9.4 \%)$ subjects in the range $30 \mathrm{e} 80 \mathrm{ng} / \mathrm{ml}(75 \mathrm{e} 200 \mathrm{nmol} / \mathrm{L})$, while154 (90.6\%) were below $30 \mathrm{ng} / \mathrm{ml}(75 \mathrm{nmol} / \mathrm{L})$ [13]. In other studies, the occurrence of osteoporosis and osteopenia varies usually in patients with IDDM, type 1 diabetes patients are more risk of fractures because high risk of falls with low BMD [15]. Reviewing in adult literature on such association found that on China, 890 ladies with type 2 diabetes, post-menopausal have lower bone mineral density level and impaired bone formation may occur [16].

In our population the fracture risk in patient with growth hormone deficient accounts for $(0.8 \%)$ of the total patient with osteoporosis. Comparing to another study, Baroncelliassessedin 46 adolescents with growth hormone deficient, lumbar spine bone mineral density and the prevalence of fracture did not differ from that of healthy people [13].

\section{Renal diseases}

In our population renal disease is considered as a common cause of secondary osteoporosis. It accounts for (6.1\%) of the total patient with osteoporosis. No published studies on pediatrics osteoporosis secondary to renal diseases. Reviewing in adult literature on such associating found that, Canadian studies include 211 patients, age over 18 with chronic kidney diseases the prevalence of vertebral fracture was presented in 74 patients [17]. A systematic review of studies worldwide found the median prevalence of CKD in adults (using the MDRD formula) is $7.2 \%$, and, in those older than 64 years of age, this increases to about $30 \%$. Another study in the USA reported the prevalence of osteoporosis varied from $10-40 \%$ in general dialysis patients [17].

\section{Immobilization}

The decrease of physical activity and delayed immobilization result in reduced mechanical tension on the bones and diminished stimulation to their formation. It has an effect on bone modeling and remodelling. Our results showed that immobilization is a cause of secondary osteoporosis, in $(6.1 \%)$ of the population, there are 8 patient complaining of immobilization, three of them are suffering from Mucopolysaccharidosis, and one have a Leigh's disease, the remaining 4 have a Neuromuscular disease. Comparing to previous reported study in Europe, the prevalence of cerebral palsy is $2 / 1000$ live birth [13]. Evaluation for 525 physically disabled children in Japan, found no difference between genders in fracture prevalence [13].

\section{Drug induced}

Long term uses of glucocorticoids can cause more excessive loss of bone [18], which causes osteopenia and fractures. In our sample of population, there are $(3.8 \%)$ had $(\mathrm{SO})$ due to steroid use, the total of patients is five, two of them also used the chemo with steroid. Other studies showed the leading cause of drug-induced osteoporosis is corticosteroids, the incidence of fracture post first year of using steroids therapy is $17 \%$, and usually asymptomatic, incidence in chronic steroid-treated patients is (30-50\%).

In a German study on (90) gastrointestinal pediatric patients, where 34 of them treated with placebo and the other 53 treated with steroid, the result was nearly equal ratio of osteoporosis in placebo-treated $(12 \%)$ and steroid-treated (11\%) patients .The other study in USA, Eugene, showing Long term used of steroid medication gives rise to bone loss specially in the first 6 months, steroid-treated patients have further risk factors for bone loss and osteoporosis related to their primary causes [19].

\section{Idiopathic}

Idiopathic juvenile osteoporosis (IJO) which is an uncommon disease with rate of incidence of 1 per 100,000that is presented by difficult movement, multiple recurrent fracture, and inclusive compression fracture of the back usually in early puberty [1]. In our data, there are only 2 patients, a global Perspective study found the prevalence of (IJO) by estimated 0.07 and 4.01 per 1000 children [13].

\section{Conclusion}

In osteoporosis the management side of bone health is very important. These involved increasing the physical activity, providing appropriate ingestion of calcium and vitamin $\mathrm{D}$, decrease intake of harmful treatments, supervision the children in the home and school to reduce exposure to accidents, also we must establish educational campaigns for osteoporosis and its complications. We conclude that osteoporosis is a widely prevalent disease in pediatric population. Therefore, we recommend that children and adolescents with various underlying causes to be screen regularly by their treating physician for associated osteoporosis as currently not highly abbreviated by most of pediatrician.

\section{References}

1. Khoshhal K (2011) Childhood osteoporosis. Journal of Taibah University Medical Sciences 6: 61-76.

2. Simm PJ, Johannesen J, Briody J, McQuade M, Hsu B, et al. (2011) Zoledronic acid improves bone mineral density, reduces bone turnover 
Citation: Abdulmoein E Al-Agha1, Alnajjar FN and Alkharji TM (2016) Causes of Osteoporosis in Pediatric Age Group Patients, Following at King Abdul Aziz University Hospital, Jeddah, Saudi Arabia: 12 Years' Experience. J Pat Care 2: 109. doi:10.4172/2573-4598.1000109

Page 5 of 5

and improves skeletal architecture over 2 years of treatment in children with secondary osteoporosis. Bone 49: 939-943.

3. Sykes B, Ogilvie D, Wordsworth P, Wallis G, Mathew C, et al. (1990) Consistent linkage of dominantly inherited osteogenesis imperfecta to the type I collagen loci: COL1A1 and COL1A2. Am J Hum Genet 46: 293-307.

4. Ma NS, Gordon CM (2012) Pediatric osteoporosis: where are we now? J Pediatr 161: 983-990.

5. Bianchi ML (2007) Osteoporosis in children and adolescents. Bone 41: 486-495.

6. Zhang C, Liu Z, Klein GL (2012) Overview of pediatric bone problems and related osteoporosis. J Musculoskelet Neuronal Interact 12: 174-182.

7. Mäkitie O (2013) Causes, mechanisms and management of paediatric osteoporosis. Nat Rev Rheumatol 9: 465-475.

8. Saag KG, Emkey R, Schnitzer TJ, Brown JP, Hawkins F, et al. (1998) Alendronate for the prevention and treatment of glucocorticoid-induced osteoporosis. Glucocorticoid-Induced Osteoporosis Intervention Study Group. N Engl J Med 339: 292-299.

9. D. N. Children's Hospital Boston: Growth Calculator 2: 1997-1999.

10. Hamill PV, Drizd TA, Johnson CL, Reed RB, Roche AF, et al. (1979) Physical growth: National Center for Health Statistics percentiles. Am J Clin Nutr 32: 607-629.

11. Patel R, Nagamani S, Cuthbertson D, Campeau P, Krischer J, et al. (2014) A cross sectional multicenter study of osteogenesis imperfecta in North
America-results from the linked clinical research centers. Clinical genetics.

12. Toumba , Skordis N (2010) Osteoporosis syndrome in thalassaemia major: an overview. J Osteoporos 2010: 537673.

13. Bianchi ML, Glorieux FH (2012) The spectrum of pediatric osteoporosis. Pediatric bone: biology and diseases: Elsevier/Academic Press Amsterdam 439-510.

14. Bikle D (2001) Osteoporosis in gastrointestinal, pancreatic, and hepatic diseases. Osteoporosis. 2: 237-58

15. Räkel A, Sheehy O, Rahme E, LeLorier J (2008) Osteoporosis among patients with type 1 and type 2 diabetes. Diabetes Metab 34: 193-205.

16. Zhou Y, Li Y, Zhang D, Wang J, Yang H (2010) Prevalence and predictors of osteopenia and osteoporosis in postmenopausal Chinese women with type 2 diabetes. Diabetes Res Clin Pract 90: 261-269.

17. Ott SM, Elder G (2013) Osteoporosis Associated with Chronic Kidney Disease. In: Cauley RMFWDLA, editor. Osteoporosis (Fourth Edition) Academic Press, San Diego 1387-1424.

18. Bockman RS, Weinerman SA (1990) Steroid-induced osteoporosis. Orthop Clin North Am 21: 97-107.

19. Boling EP (2004) Secondary osteoporosis: underlying disease and the risk for glucocorticoid-induced osteoporosis. Clin Ther 26: 1-14. 